\title{
Personalized analysis of minimal residual cancer cells in peritoneal lavage fluid predicts peritoneal dissemination of gastric cancer
}

\section{Dongbin Zhao}

Chinese Academy of Medical Sciences and Peking Union Medical College

\section{Pinli Yue}

Chinese Academy of Medical Sciences and Peking Union Medical College

\section{Tongbo Wang}

National Cancer Center/National Clinical Research Center for Cancer/Cancer Hospital, Chinese Academy of Medical Sciences and Peking Union Medical College

\section{Pei Wang}

Chinese Academy of Medical Sciences and Peking Union Medical College

\section{Qianqian Song}

Chinese Academy of Medical Sciences and Peking Union Medical College

\section{Jingjing Wang}

Jinchenjunchuang Clinical Laboratory

Yuchen jiao ( $\sim$ jiaoyuchen@126.com )

Chinese Academy of Medical Sciences and Peking Union Medical College https://orcid.org/0000-00017917-8810

\section{Article}

Keywords: Gastric Cancer, Peritoneal lavage fluid, Peritoneal dissemination, Personalized mutation assay, Minimal residual disease

Posted Date: March 10th, 2021

DOI: https://doi.org/10.21203/rs.3.rs-275520/v1

License: (a) (i) This work is licensed under a Creative Commons Attribution 4.0 International License.

Read Full License 
Author information

Dongbing Zhao,, ${ }^{1,4}$ Pinli Yue, ${ }^{2,4}$ Tongbo Wang, ${ }^{1,4}$ Pei Wang, ${ }^{2}$ Qianqian Song, ${ }^{2}$ Jingjing Wang, ${ }^{3}$ Yuchen Jiao ${ }^{2}$

Author Affiliations

${ }^{1}$ Department of Pancreatic and Gastric Surgical Oncology, National Cancer Center/National Clinical Research Center for Cancer/Cancer Hospital, Chinese Academy of Medical Sciences and Peking Union Medical College, Beijing, 100021, China. ${ }^{2}$ State Key Laboratory of Molecular Oncology, National Cancer Center/National Clinical Research Center for Cancer/Cancer Hospital, Chinese Academy of Medical Sciences and Peking Union Medical College, Beijing, 100021, China. ${ }^{3}$ Jinchenjunchuang Clinical Laboratory, Hangzhou, Zhejiang, China. ${ }^{4}$ These authors contributed equally: Dongbing Zhao, Pinli Yue and Tongbo Wang. (5)

Address correspondence to: Yuchen Jiao, Cancer Hospital, Chinese Academy of Medical Sciences and Peking Union Medical College, Panjiayuan nanli 17, Chaoyang District, Beijing 100021, China. Phone: 86.10.87787662; E-mail: ¡iaoyuchen@cicams.ac.cn 


\section{ABSTRACT}

Peritoneal dissemination (PD) is the major type of gastric cancer $(\mathrm{GC})$ recurrence and leads to rapid death. Current approach cannot precisely determine which patients are at high risk of PD. In this study, we developed a technology to detect minimal residual cancer cells in peritoneal lavage fluid (PLF) samples by parallel profiling tumor-specific mutations. We applied the technology to a prospective cohort of $110 \mathrm{GC}$ patients. The technology showed ultra-high sensitivity by successfully detecting all the 27 cases that developed PD. The minimal residual cancer cells in PLF was associated with an increased risk of PD $(H R=145.13 ; 95 \% \mathrm{Cl}=$ 20.20-18435.79; $p<0.001$ ) and significantly shorter overall survival. In pathologically high-risk (T4) patients, the PLF mutation profiling model exhibited even greater specificity of $91 \%$ and positive predictive value of $88 \%$, while retaining sensitivity of $100 \%$. PLF cancer cell fraction remained the strongest independent predictor of PD and recurrence-free survival over pathologic diagnosis and cytological diagnosis in GC patients. This approach may help in the postsurgical management of GC patients by detecting PD far before the metastatic lesions grow to significant size detectable by conventional methods such as MRI and CT scanning.

Key words: Gastric Cancer; Peritoneal lavage fluid; Peritoneal dissemination; Personalized mutation assay; Minimal residual disease 


\section{INTRODUCTION}

Gastric Cancer (GC) is the fifth most common and the third leading cause of cancer death in the world ${ }^{1}$. Peritoneal dissemination (PD) is the most common form of GC recurrence and a strong indicator of poor prognosis with a $13 \%$ overall 5 -year survival rate ${ }^{2}$. Although multiple therapeutic solutions, such as hyperthermic intraperitoneal chemotherapy (HIPEC) and extensive intraoperative peritoneal lavage plus intraperitoneal chemotherapy (EIPL-IPC), have been developed as prophylactic strategies targeting $\mathrm{PD}^{3,4}$, the preventive effect is compromised due to the difficulty to precisely identify the patients who will develop PD. Many studies have demonstrated that HIPEC and IPC could effectively prevent PD and improve the survival rate of patients with advanced gastric cancer ${ }^{5-7}$. However, once the peritoneal metastasis reaches a stage detectable by imaging, subsequent chemotherapy is not effective because of the complex intraperitoneal environment, and the advanced PD leads to many complications and rapid death (5-16 months $)^{8-11}$. Thus, prediction or early detection of PD would help identify the GC patients who need intensive therapy to prevent PD.

Several studies shed light on the prediction of PD by the detection of cancer cells in peritoneal lavage fluid (PLF) samples ${ }^{12}$. Previous studies of peritoneal metastasis focused on conventional cytology. However, the sensitivity to detect minimal residual cancer cells to predict PD reached no more than $30 \%{ }^{13-15}$. With the development of reverse transcriptase PCR (RT-PCR) technology, several studies began to examine cancer tissue specific messenger RNAs (mRNA) in PLF, such as carcinoembryonic antigen (CEA $)^{16-18}$, cytokeratin-20 $(\mathrm{CK}-20)^{19}$, and matrix metalloproteinase-7 (MMP-7) ${ }^{20}$. None of these mRNAs is an ideal marker due to low sensitivity or specificity. The main source of such results is thought 
to be the amplification of low-level CEA from peritoneal inflammatory cells, and the aberrant expression of mRNA originating from granulocytes ${ }^{21}$. In recent years, there have been several minimal residual disease (MRD) studies using exosomes and methylation to predict $\mathrm{PD}^{22,23}$. Some studies detected MRD by profiling GC-specific methylation alterations with bisulfite treatment and $\mathrm{PPCR}^{24,25}$. The clinical application of such MRD detection from PLF is limited primarily for two reasons. First, GC is a highly heterogeneous disease, rendering it difficult to identify highly tumor-specific and prevalent biomarkers among all GC cases. Second, the ratio of residual cancer cells among normal cells in PLF can be very low and undetectable with standard qPCR-based methods.

Recent developments in next generation sequencing (NGS) enable genome-wide profiling of tumor tissues to identify somatic mutations which are highly tumor-specific biomarkers to track $M R D^{26}$. With recent developments in experimental and bioinformatic technologies, high throughput sequencing can also be applied to detect low frequency mutations in cell free DNA (cfDNA), providing a basis to improve MRD detection with high sensitivity ${ }^{27}$. Several recent studies have profiled tumor samples for somatic mutations and tracked the mutations in the cfDNA from matched blood samples to detect $M R D^{28-31}$. The mutation-based MRD detection showed strong performance in the prediction of recurrence in multiple tumor types.

In this study, we applied Mutation Capsule, a mutation profiling technology, to profile up to 20 mutations on the genomic DNA of cell pellets of PLF samples collected after abdominal exploration and before any manipulation of the stomach. We developed a model to evaluate the fraction of cancer cells among normal cells based on the number, fraction and sequencing 
depth of the mutations detected. The assay precisely predicted all PD cases with $100 \%$ sensitivity, $85 \%$ specificity, $71 \%$ positive predictive value (PPV) and $100 \%$ negative predictive

87 value (NPV). For stage T4 GC patients, the approach exhibited higher specificity (91\%) and PPV (88\%), with sensitivity and NPV at 100\%. assay to profile MRD in cells pelleted from PLF. Our study demonstrates that PLF cancer cell

91 fraction is a powerful biomarker for prognosis and early detection of PD.

92 


\section{RESULTS}

\section{Patient enrollment and study design}

A total of 110 patients with stages I to III GC (mean [SD] age, 61 [9.6] years; 79 [76\%] male) were enrolled in the study. Six patients were excluded from the study for palliative surgery $(n=$ 4 ) or the presence of other types of cancer $(n=2)$. Finally, a total of 104 patients were included in the analysis (Fig. 1). Recurrence of PD was defined as lesions detected in one of the following ways: MRI, CT scan, PET-CT scan visualized $\left[{ }^{18} \mathrm{~F}\right]$ fluorodeoxyglucose $\left({ }^{18} \mathrm{~F}-\mathrm{FDG}\right)$ uptake over peritoneum and bowel with or without ascites $>50 \mathrm{~mL}$, or tumor cells detected in ascites or biopsy of peritoneal lesions. The median follow-up period was 20 months (6-41 months). Clinicopathological characteristics of the 104 patients are listed in Supplementary Table 1. Surgery consisted of a radical resection of the primary tumor and at least D2 lymph node dissection.

We performed exome sequencing on the DNA from tumor tissue and matched white blood cell (WBC) samples to identify somatic mutations. A median of 101 mutations were detected in the primary tumor samples of each patient. We designed customized primers to profile up to 20 somatic mutations in the peritoneal lavage samples with the Mutation Capsule technology (Supplementary Table 2) ${ }^{32}$.

\section{Construction and validation of the cancer cell fraction model}

Due to the low fraction of residual cancer cells among normal cells in PLF, the number of molecules present for a given mutation site in the sequencing (distinct coverage) might be insufficient to precisely detect the mutation. Thus, the detected frequency of one mutation 
might not precisely represent the ratio of cancer cells ${ }^{33,34}$. Here, we set up a model to estimate the cancer cell ratio based on allele frequency and sequencing depth of somatic mutations in tumor tissue and paired PLF samples (Fig. 2a). For each traced somatic mutation, we determined the mutation frequency in the PLF sample and compared with the frequency in the primary tumor. The sample-level estimated cancer cell fraction was determined with the Maximum Likelihood Estimation.

To validate the accuracy of the cancer cell fraction model, we serially diluted cells from the cell line PLC/PRF/5 with cells from a second cell line A549 to generate 9 dilutions (PLC/PRF/5 cell fraction $=0 \%, 0.0001 \%, 0.0003 \%, 0.001 \%, 0.005 \%, 0.05 \%, 0.5 \%, 5 \%$ and $33 \%)$. We selected 20 unique SNPs in PLC/PRF/5, and designed a customized assay targeting the SNPs. In each diluted sample, we profiled the 20 SNPs to calculate the estimated dilution ratio with the model (Supplementary Table 3). The data exhibited a strong linear correlation between the theoretical and estimated dilution ratios up to the dilution of $1: 100,000\left(R^{2}=\right.$ 0.9998) (Fig. 2b). Background noise observed at $0 \%$ PLC/PRF/5 cell input was $0.0007 \%$ (maximum value) among the 20 independent replicates (Fig. 2c). Based on the analytical validation results, the cancer cell fraction model exhibited $100 \%$ sensitivity at $0.001 \%$ spiked-in cell fraction, $67 \%$ sensitivity at $0.0003 \%$ cell fraction, and $33 \%$ sensitivity at $0.0001 \%$ cell fraction (Supplementary Table 4 and Supplementary Fig. 1). The data indicated a limit of detection (LOD) at a $0.001 \%$ cancer cell fraction based on the multi target site model.

To evaluate the biological noise of random mutations from non-tumor cells in PLF samples, we determined the fraction of 20 mutations that were not detected in the matched tumor sample. We calculated a set of cancer cell fractions based on these non-tumor-specific 
mutations, and found the fractions from background noise in all PLF samples to be lower than $0.01 \%$ (Fig. 2d, mean $=0.0006 \%$, maximum $=0.0082 \%$ ). To achieve a high specificity, we classified the cancer cell fraction as positive or negative with a cutoff of $0.01 \%$ in further analyses.

Association of peritoneal lavage cancer cell fraction with risk of peritoneal dissemination

For the 104 patients, we chose a total of 1,717 mutations in the analysis (Supplementary Table 5). On average, 17 somatic mutations (3-23 mutations per person) were tracked for each patient (Fig. 3a), and $25.7 \%(441 / 1,717)$ of the tracked mutations were detected in matched PLF samples. We calculated the cancer cell fraction based on the frequency and distribution of the tracked mutations with the model. The cancer cell fraction ranged from 0 to $23.41 \%$ (mean $0.48 \%$, median $0.0074 \%)$. Forty-two out of the $104(40 \%)$ patients were positive $(\geq 0.01 \%$ cancer cell fraction in PLF sample), and 62 (60\%) were negative in the PLF mutation profiling model (Fig. 3a and Supplementary Table 6). Among the 104 patients, 27 patients experienced peritoneal dissemination, and all $(27 / 27,100 \%)$ of them tested positive in the PLF mutation profiling model. Six patients developed lymphatic metastasis, and 4 (67\%) tested positive in the PLF mutation profiling model. In addition, 15\% (11/71) of the non-recurrence patients were positive. The differences in cancer cell fraction between recurrence and non-recurrence groups were significant (PD, mean [SD], 0.0169 [0.04706] versus non-recurrence, mean [SD], 0.0004 [0.00294], $p<0.001$; lymphatic metastasis, mean [SD], 0.0020 [0.00433] versus non-recurrence, mean [SD], 0.0004 [0.00294], $p=0.013$ ) (Fig. 3b). 
of $\mathrm{PD}(\mathrm{HR}=145.13 ; 95 \% \mathrm{Cl}=20.20-18435.79 ; p<0.001)$ (Fig. 4a). Twenty-seven of the 38

$161(71 \%)$ test-positive patients and none of the $60(0 \%)$ test-negative patients experienced PD.

Kaplan-Meier estimates of recurrence free interval (RFI) at 3 years for test-positive patients

163

164

165

166

167

were $17.60 \%(95 \% \mathrm{Cl}=0 \%-35.24 \%)$, and for test-negative patients, $100 \%$. In receiver operating characteristic $(\mathrm{ROC})$ analysis, cancer cell fraction (AUC $=0.92$ ) achieved $100 \%$ sensitivity and $85 \%$ specificity, with a PPV of $71 \%$ (Table 1$)$

\section{Comparison of the cancer cell fraction and clinical risk factors on the prediction of PD}

We further compared the predictive value of the PLF mutation profiling model with clinical risk factors including cytological diagnosis and pathologic diagnosis, which was defined as high (T4) or low (T0-3) according to the standard criteria. The RFI outcomes of cytological and pathologic diagnosis risk assessments were the following, respectively: $\mathrm{HR}=4.92 ; 95 \% \mathrm{Cl}=$ 2.10-11.51; $p<0.001$ (Supplementary Fig. $2 \mathrm{a}$ ); and $\mathrm{HR}=5.04 ; 95 \% \mathrm{Cl}=1.74-14.58 ; p=0.001$ (Supplementary Fig. 2b). Cytological diagnosis exhibited high specificity $(94 \%, 67 / 71)$ but low sensitivity $(30 \%, 8 / 27)$. On the other hand, pathologic diagnosis showed high sensitivity $(85 \%$, $23 / 27)$ but low specificity $(54 \%, 38 / 71)$ (Table 1$)$. The performance of cytological diagnosis and pathologic diagnosis were consistent with previous reports ${ }^{35-37}$.

Interestingly, we found that 8 of the 11 false positive cases by the PLF mutation profiling model occurred in stage T1-3 cases, while only 4 cases in this group developed PD. Among the pathologically high-risk patients of stage T4 $(n=56)$, the PLF mutation profiling model exhibited a lower false positive rate, delivering 100\% (23/23) sensitivity, 91\% (30/33) specificity, 
181

and $88 \%$ (23/26) PPV. The RFI outcomes of PLF cancer cell fraction assessment in stage T4 cases was $\mathrm{HR}=132.43 ; 95 \% \mathrm{Cl}=17.55-17006.37 ; p<0.001$ (Fig. $4 \mathrm{~b}$ ).

To adjust for multiple variables in a single model, we used a cox proportional hazard model. PLF cancer cell fraction remained the strongest independent predictor of recurrence-free survival $(\mathrm{RFS})(\mathrm{HR}=141.27,95 \% \mathrm{Cl}=17.37-18633.39 ; p<0.001)$, followed by pathologic diagnosis $(\mathrm{HR}=3.82,95 \% \mathrm{Cl}=1.21-17.27 ; p=0.02)$ (Supplementary Table 7).

\section{Prediction of recurrence with the PLF mutation profiling model}

Six of the 104 patients experienced lymphatic metastasis during the 41-month follow-up. Of these 6 samples, 4 were positive in the PLF mutation profiling test. When we combined peritoneal dissemination and lymphatic metastasis patients as a recurrence group, the test-positive patients by the PLF mutation profiling model were 40 times more likely to relapse than the test-negative patients $(\mathrm{HR}=39.97 ; 95 \% \mathrm{Cl}=9.50-168.10 ; p<0.001)$ (Supplementary Fig. 2c). Kaplan-Meier estimates of RFI at 3 years for test-positive patients was $15.80 \%$ (95\% $\mathrm{Cl}=0 \%-31.68 \%)$, and for those test-negative was $96.50 \%(95 \% \mathrm{Cl}=91.80 \%-100 \%)$. The PLF mutation profiling model achieved $94 \%$ (31/33) sensitivity, 85\% (60/71) specificity, 74\% (31/42) PPV and 97\% (60/62) NPV (Table 1).

During the follow-up period, 10 patients had developed disease recurrence and died from metastatic disease, and all of them were test-positive in the PLF mutation profiling model. A significantly shorter overall survival (OS) was associated with a positive result in the model $(\mathrm{HR}=50.35 ; 95 \% \mathrm{Cl}=6.41-6492.15 ; p<0.001)($ Figure $4 \mathrm{C})$. The median survival of these 10 patients was 22 months (6-39 months). 


\section{DISCUSSION}

PD accounts for $50 \%$ of recurrence in GC patients and is associated with exceptionally poor prognosis $^{38}$. CT and other imaging modalities are used to diagnose PD, but the sensitivity to predict or detect PD in early and curable stage is inadequate as such metastatic lesions could be small and easily missed. When GC patients show PD-associated symptoms such as ascites and intestinal obstruction, or when metastases can be observed by imaging, the PD lesions are often in significant size and advanced stage, with no effective treatments available. In addition to peritoneal cytology, multiple biomarkers including mRNA, methylation and protein markers have been used to profile PLF to predict PD among GC patients ${ }^{39-41}$. However, the accuracy of these approaches for the early detection of PD is still limited ${ }^{13,42}$. A potential reason is that GC is a highly heterogeneous disease, rendering it difficult to find a marker suitable for all GC cases. In addition, the fraction of cancer cells among PLF cell pellets could be very low and beyond the limit of detection.

MRD detection based on a customized assay targeting tumor-specific mutations in plasma cfDNA have shown promising performance in prognostic prediction and disease monitoring in several tumor types, including breast, colorectal and lung cancers ${ }^{28,30,43}$. Other non-blood samples have also been used for specific clinical scenarios. For example, cell pellets in urine have been used to monitor the development of bladder cancers ${ }^{44}$. In this study, we developed a customized assay to profile up to 20 tumor-specific mutations in PLF DNA. We also set up a model to evaluate the fraction of cancer cells. Using a standard reference of mixed cancer cell lines, we found that the approach confidently detected cancer cells when the fraction was as low as $0.001 \%$. In clinical PLF samples, the biological noise was close to 

predicting PD at the time of surgery, especially in stage T4 patients.

$0.01 \%$, possibly due to random mutations in epithelial cells or white blood cells ${ }^{45}$. predict the development of PD. An interventional clinical trial targeting T4 and PLF-mutation-positive patients might answer the question as to whether intensive survival.

We applied the pipeline to detect MRD in PLF samples from GC patients. The approach exhibited a strong performance in the prediction of PD with $100 \%$ sensitivity, $85 \%$ specificity and $71 \%$ PPV. The sensitivity of the mutation profiling model is higher than that of peritoneal cytology or pathology based on a direct comparison in the same cohort. Mutation profiling also exhibited increased performance relative to other previously reported PLF-based detection assays $^{46-48}$. Furthermore, the combination with clinical risk factors (stage T4) further improved the performance. In the subgroup of T4 high risk individuals, the mutation profiling model showed an even stronger performance with $100 \%$ sensitivity, $91 \%$ specificity and $88 \%$ PPV. Therefore, the PLF mutation profiling should be especially helpful for stage T4 patients to post-operational treatment targeting PD might help to prevent recurrence and improve overall

Our study suggests that mutation-based MRD profiling in PLF samples is effective in 241 


\section{METHODS}

Patients and samples

244 From June, 2017, to December, 2019, 110 gastric cancer patients scheduled for surgery in National Cancer Center, Cancer Hospital, Chinese Academy of Medical Sciences, were enrolled in our study. Eligibility criteria of the study included a diagnosis of stage I to III resectable gastric cancer determined by chest-abdominal-pelvic enhanced computed tomography-scan (CT-scan), MRI or upper gastrointestinal endoscopy \pm endoscopic ultrasound (EUS); no metastatic disease evident on staging computed tomography (CT); age $<80$ years; no preoperative radiotherapy or chemotherapy; and a treatment plan for resection. In accordance with NCCN guidelines, postoperative chemotherapy was recommended after surgery. Patients with another malignant neoplasm diagnosed within the last 3 years were excluded. After therapy, surveillance was performed according to the standard of care, with clinical assessment every 3 months for the first 2 years and every 6 months for the next years. TNM stages were determined according to the 8th edition of the Union for International Cancer Control.

For collection of PLF samples, $300-400 \mathrm{~mL}$ of normal saline was used to wash the upper abdominal cavity after abdominal exploration and before any manipulation of the tumor during surgery. A total of $200 \mathrm{~mL}$ of PLF was collected. PLF (100 mL) was examined through conventional cytological diagnosis with Papanicolaou and Giemsa staining. The remaining 100 $\mathrm{mL}$ of PLF was used in mutation profiling. PLF samples were centrifuged first at $2500 \mathrm{rpm}$ (1098g) for $10 \mathrm{~min}$ to remove the supernatant, and further centrifuged at $12,000 \mathrm{rpm}$ for $10 \mathrm{~min}$ 
blood draw protocol. Fresh frozen tumor tissue was collected from surgery. Tumor tissues, matched white blood cells (WBC) and PLF pellets were stored at $-80^{\circ} \mathrm{C}$ until DNA extraction. In total, PLF, frozen tumor tissue and blood samples were collected for analysis from 104 patients.

\section{Cell lines and standard reference}

The two cancer cell lines hepatocarcinoma PLC/PRF/5 cells and lung carcinoma A549 cells used to construct the cancer cell fraction model were both obtained from the Cell Bank of the Chinese Academy of Sciences (Shanghai, China). In the model, PLC cells were used as the "tumor cells" and diluted with A549 cells which were used as the "normal cells". The two cell lines were counted with FACSAriaSORP flow cytometry (Becton Dickinson; San Diego, CA,USA) and serially diluted for a total of 9 dilutions (PLC cell fraction $=0 \%, 0.0001 \%$, $0.0003 \%, 0.001 \%, 0.005 \%, 0.05 \%, 0.5 \%, 5 \%$ and $33 \%)$. The details of the model construction and number of cells are shown in Supplementary Table 4.

\section{DNA extraction and library preparation}

Genomic DNAs were extracted from PLF, frozen tumor tissue, WBC and cell line samples using the QIAamp DNA Mini Kit (Qiagen; Hilden, Germany). Exome sequencing was performed on genomic DNA (1000 ng) from frozen tumor tissue and WBC, and customized panel targeting 20 mutations was performed on genomic DNA (200 ng) from cell lines or PLF. Genomic DNA was sheared into small fragments (mean length $200 \mathrm{bp}$ ) with the Covaris E220 instrument (Covaris; Woburn, MA, USA). The sheared DNA was prepared for genomic 
libraries using the KAPA Hyper Prep Kit (Roche; Basel, Switzerland) through a series of enzymatic steps including, end-repair, dA tailing, ligation of adaptors and amplification following standard protocols except that for the use of a customized adaptor with barcodes for PLF and cell line samples as previously described ${ }^{32,49}$.

\section{Whole-exome sequencing and identification of somatic mutations}

Whole genome libraries of tumor and matched WBC DNAs were enriched for exome regions with Agilent SureSelectXT Human All Exon V5 probe and reagents (Agilent; Santa Clara, CA, USA). The captured and amplified libraries were sequenced on the Illumina HiSeqX Ten with 150-bp paired-end sequencing to a median depth of $183 x$ for tumor tissue samples and $105 x$ for WBC samples after removing duplicate molecules. The sequencing raw data (FASTQ file) were aligned to the UCSC human reference genome hg19 using the Burrows-Wheeler aligner software (BWA, v0.7.15). Basic processing, marking duplicates, local realignments and score recalibration were analyzed using The Genome Analysis Toolkit (GATK, v3.6), Picard (v2.7.1) and Samtools (v1.3.1). Candidate somatic mutations were detected by comparing sequencing data from tumor tissue samples with MuTect1 and Strelka. All selected mutations were further validated with manual inspection using Integrated Genome Viewer (IGV) ${ }^{50}$.

\section{Targeted sequencing in cell line samples}

The whole genome libraries generated from cell line DNA were captured using a 63-gene panel (Supplementary Table 8, $202 \mathrm{~kb}$ ) and Agilent SureSelect hybrid capture reagents. The sequencing and analysis flow of the captured library is consistent with that of whole exome 
sequencing (WES). PLC cells were used as the tumor sample and A549 cells were used as the normal cell sample, with a median depth of $8221 \times$ distinct high quality reads for PLC samples and $6721 \times$ for $A 549$ samples. 20 SNPs specific for PLC were chosen and compared with A459. These SNPs were assessed in the diluted cell line samples where the mutation frequency was $100 \%$ in the PLC cell line and $0 \%$ in the A549 cell line using the previously described method ${ }^{32}$. Experiments were repeated three times on samples from each dilution and 20 times for the 0\% PLC cell fraction. Targeted sequencing achieved median pre-deduplication sequencing depths of $415,165 \times$ and $20,074 \times$ after removing duplicate molecules for each detection SNP.

\section{Customized assay to profile multiple mutations}

Genomic DNAs (200 ng) from cell lines or PLF were used for the customized panel targeting 20 mutations. The ligation products with customized adaptor were amplified for a whole genome library and used as template to profile mutations identified in the matched tumor tissue. The customized adaptor contained sufficient unique DNA barcodes identifying each original molecule ${ }^{32}$. We selected 20 tumor-specific somatic mutations from the WES results of tumor tissue for primer design. For each patient, the 20 tumor-specific variants were selected according to the potential to be a driver mutation (Supplementary Table 9), the confidence of the mutation by IGV and the frequency of the mutation. Oligo software (v7.53) was used to design multiplex-PCR primer pairs for the two rounds of nested amplification and verified uniqueness in the human genome (http://genome.ucsc.edu/) to ensure amplification efficiency. In the first round of amplification, the target regions were amplified in 9 cycles of PCR using a 
target-specific primer and a primer matching the adapter sequence. A second round of 14 cycles of amplification was performed with one pair of nested primers matching the adapter and the target region to further enrich the target region and add the Illumina adapter sequences to the construct. This method can amplify more than 200 target regions in parallel. The targeted sequencing libraries were sequenced on the Illumina Novaseq 6000 with a median depth of $101,703 \times$ before de-duplication and $11,760 \times$ after removing duplicate molecules. The on-target ratio of reads mapped to the target region was $80 \%$ in median.

\section{Bioinformatics pipeline and SNV calling}

With the DNA barcode in the customized adapter, redundant reads can be tracked from an original DNA molecule to minimize false positive calling due to PCR amplification and sequencing errors. Sequencing reads were mapped to the hg19 reference genome using 'bwa mem' with the default parameters after extracting tags and removing sequence adapters. The SNV analysis mutation frequency calculation method was used as previously described ${ }^{32}$. Briefly, reads with the same tags and start and end coordinates were grouped into Unique Identifier families (UID families). If $>80 \%$ of the reads in a UID family harbor the same mutation identified in the matched tumor tissue, the UID family was defined as an Effective Unique Identifier family (EUID family) with the mutation. The mutant EUID families were further confirmed by manual inspection (IGV) and used to calculate the frequency of the mutation with the total number of UID families covering the mutant site.

\section{PLF mutation profiling model}


352

353

354

binomial distribution:

$$
X_{i} \sim B\left(D_{i}, P c_{i}\right)
$$
is:

$$
P_{i}\left(X_{i}=A_{i}\right)=\left(\begin{array}{c}
D_{i} \\
A_{i}
\end{array}\right) P c_{i}^{A_{i}}\left(1-P c_{i}\right)^{D_{i}-A_{i}}
$$

Assuming that the events of observing $\left(A_{1}, A_{2}, \ldots, A_{n-1}, A_{n}\right)$ reads supports that mutations $\left(m_{1}, m_{2}, \ldots, m_{n-1}, m_{n}\right)$ are independent, then the probability of observing a sequence of $\left(A_{1}, A_{2}, \ldots, A_{n-1}, A_{n}\right)$ is the product of $P_{i}\left(X_{i}=A_{i}\right)$ for each mutation $m_{i}$. Let $P$ be the

Estimated cancer cell fraction analysis was performed in the $\mathrm{R}$ statistical environment version 3.6.3.

Assumption: 1. Because of the low fraction of residual cancer cells among normal cells in PLF, there are chances that some mutations present in the tumor tissue are not detected in the corresponding PLF sample. The algorithm is also based on the assumption that this tumor-PLF mismatch results from the low concentration of mutant molecules and/or the randomness in sampling. 2. The estimation model is based on the assumption that mutant allele reads and non-mutant wild-type allele reads fit well with the binomial distribution.

Let $\left(m_{1}, m_{2}, \ldots, m_{n-1}, m_{n}\right)$ be $\mathrm{N}$ mutations in PLF, each with a mutation frequency of $P c_{i}$, sequencing depth of $D_{i}$, mutation reads number of $A_{i}$. And $P t_{i}$ indicates the mutation Then the probability of observing $A_{i}$ reads with mutation $m_{i}$ out of $D_{i}$ reads at position $m_{i}$

likelihood function of cancer cell concentration $R$ : 


$$
L(R)=P=\prod_{i=1}^{n} P_{i}=\prod_{i=1}^{n}\left(\begin{array}{c}
D_{i} \\
A_{i}
\end{array}\right)\left(P t_{i} * R\right)^{A_{i}}\left(1-P t_{i} * R\right)^{D_{i}-A_{i}}
$$

372 And $P$ is maximized to obtain the predicted $R$, namely $\hat{R}$ :

$$
\begin{aligned}
\hat{R} & =\underset{R}{\arg } \max _{i=1}^{n} P_{i}=\prod_{i=1}^{n}\left(\begin{array}{c}
D_{i} \\
A_{i}
\end{array}\right)\left(P t_{i} * R\right)^{A_{i}}\left(1-P t_{i} * R\right)^{D_{i}-A_{i}} \\
& =\underset{R}{\max } \ln \left[\prod_{i=1}^{n}\left(\begin{array}{c}
D_{i} \\
A_{i}
\end{array}\right)\left(P t_{i} * R\right)^{A_{i}}\left(1-P t_{i} * R\right)^{D_{i}-A_{i}}\right]
\end{aligned}
$$

373

$$
=\arg \max _{R} \sum_{i=1}^{n}\left[\ln \left(\begin{array}{c}
D_{i} \\
A_{i}
\end{array}\right)+A_{i} * \ln \left(P t_{i} * R\right)+\left(D_{i}-A_{i}\right) * \ln \left(1-P t_{i} * R\right)\right]
$$

374 A search through a grid $(0.00001,1$, step $=0.00001)$ for $R$ in the above formula yields the optimized $\hat{R}$. Maximum mutation frequencies in tumor tissues were used for normalization of tumor sample purity.

\section{Statistics}

Recurrence-free survival (RFS) was calculated as the time from surgery to the date of

recurrence, last visit or death, whichever comes first. Overall survival (OS) was measured from surgery to the date of cause-specific death or last visit. Survival curves were plotted and analyzed with the Kaplan-Meier method, and the log-rank test was used to test the significance of the difference between survival curves.

In addition to the cancer cell fraction, the prognostic impact of other factors, such as also analyzed. HRs were first estimated using univariate Cox proportional hazard models. 
388

analysis using the Cox proportional hazard model. Whenever there was no event in one group of categorical variables, Firth's penalized likelihood was adopted to allow for monotone likelihood (R package "coxphf"). All statistical analysis was performed using the survival package or coxphf package from the R software (V.3.6.3). Statistical comparison of the cancer cell fraction distribution in patients with no recurrence, peritoneal dissemination or lymphatic metastasis was performed using 2-tailed Wilcoxon Mann-Whitney $\mathrm{U}$ test with significance level set at $5 \%$ and results are expressed as mean \pm SD. $P<0.05$ was considered statistically significant.

\section{Data availability}

The sequencing data from tumor tissue, WBC and PLF samples have been deposited at the Genome Sequence Archive for Human under the accession code HRA000528 (https://bigd.big.ac.cn/gsa-human/).

\section{Study approval}

All patients provided written informed consent, and the study was approved by Ethics Committee of National Cancer Center, Cancer Hospital, Chinese Academy of Medical Sciences and Peking Union Medical College (approval number: 17-093/1349). 
407

408

409

410

411

412

413

414

415

416

417

418

419

420

421

422

423

424

425

\section{Author Contributions}

D.Z.: designed the study, organized sample collection, acquired data, interpreted data, and provided study supervision. P.Y.: performed experiments, analysed data, interpreted data, and wrote the manuscript. T.W.: Organized patient enrollment, sample collection, and clinical data curation. P.W.: developed and optimized experimental protocols, and analysed data. Q.S.: analyzed data. J.W.: interpreted data and wrote the manuscript. Y.J.: designed the study, acquired, analyzed and interpreted data, wrote the manuscript, and provided study supervision. All authors critically reviewed the manuscript.

Acknowledgments: This study was supported by National Key R\&D Program of China (2018YFC1312100), the Chinese Academy of Medical Sciences (CAMS) Innovation Fund for Medical Sciences (CIFMS) (2016-I2M-1-001, 2019-I2M-1-003 and 2017-I2M-4-002).

Correspondence and requests for materials should be addressed to Yuchen Jiao.

Conflict of interest: Y.J. is one of the cofounders and have owner interest in Genetron Holdings, and receives royalties from Genetron. Y.J., D.Z., P.W., Q.S., and P.Y. have filed patents/patent applications based on the technology and data generated from this work. The remaining authors disclose no conflicts. 
1 Bray, F. et al. Global cancer statistics 2018: GLOBOCAN estimates of incidence and mortality worldwide for 36 cancers in 185 countries. CA Cancer J Clin 68, 394-424 (2018).

2 Badgwell, B. et al. Long-term survival in patients with metastatic gastric and gastroesophageal cancer treated with surgery. J Surg Oncol 111, 875-881 (2015).

3 Kuramoto, M. et al. Extensive intraoperative peritoneal lavage as a standard prophylactic strategy for peritoneal recurrence in patients with gastric carcinoma. Ann Surg 250, 242-246 (2009).

4 Desiderio, J. et al. The 30-year experience-A meta-analysis of randomised and high-quality non-randomised studies of hyperthermic intraperitoneal chemotherapy in the treatment of gastric cancer. Eur J Cancer 79, 1-14 (2017).

5 Yonemura, Y. et al. Intraoperative chemohyperthermic peritoneal perfusion as an adjuvant to gastric cancer: final results of a randomized controlled study. Hepatogastroenterology 48, 1776-1782 (2001).

$6 \mathrm{Kim}$, J. Y. \& Bae, H. S. A controlled clinical study of serosa-invasive gastric carcinoma patients who underwent surgery plus intraperitoneal hyperthermo-chemo-perfusion (IHCP). Gastric Cancer 4, 27-33 (2001).

7 Roviello, F., Caruso, S., Neri, A. \& Marrelli, D. Treatment and prevention of peritoneal carcinomatosis from gastric cancer by cytoreductive surgery and hyperthermic intraperitoneal chemotherapy: overview and rationale. Eur J Surg Oncol 39, 1309-1316 (2013).

8 Gill, R. S. et al. Treatment of gastric cancer with peritoneal carcinomatosis by cytoreductive surgery and HIPEC: a systematic review of survival, mortality, and morbidity. J Surg Oncol 104, 692-698 (2011).

9 Mizrak Kaya, D. et al. Risk of peritoneal metastases in patients who had negative peritoneal staging and received therapy for localized gastric adenocarcinoma. J Surg Oncol 117, 678-684 (2018).

10 Chen, Y. et al. Predicting Peritoneal Dissemination of Gastric Cancer in the Era of Precision Medicine: Molecular Characterization and Biomarkers. Cancers (Basel) 12 (2020).

11 Yang, X. J., Li, Y. \& Yonemura, Y. Cytoreductive surgery plus hyperthermic intraperitoneal chemotherapy to treat gastric cancer with ascites and/or peritoneal carcinomatosis: Results from a Chinese center. J Surg Oncol 101, 457-464 (2010).

12 Lisiecki, R., Kruszwicka, M., Spychała, A. \& Murawa, D. Prognostic significance, diagnosis and treatment in patients with gastric cancer and positive peritoneal washings. A review of the literature. Rep Pract Oncol Radiother 22, 434-440 (2017).

13 Wang, J.-Y. et al. Gastric cancer cell detection in peritoneal lavage: RT-PCR for carcinoembryonic antigen transcripts versus the combined cytology with peritoneal carcinoembryonic antigen levels. Cancer Letters 223, 129-135 (2005).

14 Yonemura, Y. et al. Inhibition of peritoneal dissemination in human gastric cancer by MMP-7-specific antisense oligonucleotide. J Exp Clin Cancer Res 20, 205-212 (2001).

15 Tokuda, K. et al. Clinical significance of CEA-mRNA expression in peritoneal lavage fluid from patients with gastric cancer. Int J Mol Med 11, 79-84 (2003).

16 Kodera, Y. et al. Prognostic significance of intraperitoneal cancer cells in gastric carcinoma: analysis of real time reverse transcriptase-polymerase chain reaction after 5 years of followup. J Am Coll Surg 202, 231-236 (2006).

17 Nakanishi, H. et al. Rapid quantitative detection of carcinoembryonic antigen-expressing free 
tumor cells in the peritoneal cavity of gastric-cancer patients with real-time RT-PCR on the lightcycler. Int J Cancer 89, 411-417 (2000).

18 Kodera, Y. et al. Quantitative detection of disseminated free cancer cells in peritoneal washes with real-time reverse transcriptase-polymerase chain reaction: a sensitive predictor of outcome for patients with gastric carcinoma. Ann Surg 235, 499-506 (2002).

19 Kodera, Y. et al. Prognostic significance of intraperitoneal cancer cells in gastric carcinoma: detection of cytokeratin 20 mRNA in peritoneal washes, in addition to detection of carcinoembryonic antigen. Gastric Cancer 8, 142-148 (2005).

20 Yonemura, Y. et al. Prediction of peritoneal micrometastasis by peritoneal lavaged cytology and reverse transcriptase-polymerase chain reaction for matrix metalloproteinase-7 mRNA. Clin Cancer Res 7, 1647-1653 (2001).

$21 \mathrm{Kim}, \mathrm{Y}$. J. et al. The Detection of Messenger RNA for Carcinoembryonic Antigen and Cytokeratin 20 in Peritoneal Washing Fluid in Patients with Advanced Gastric Cancer. Korean J Gastroenterol 69, 220-225 (2017).

22 Tokuhisa, M. et al. Exosomal miRNAs from Peritoneum Lavage Fluid as Potential Prognostic Biomarkers of Peritoneal Metastasis in Gastric Cancer. PLoS One 10, e0130472 (2015).

23 Ohzawa, H. et al. Reduced expression of exosomal miR-29s in peritoneal fluid is a useful predictor of peritoneal recurrence after curative resection of gastric cancer with serosal involvement. Oncol Rep 43, 1081-1088 (2020).

24 Hiraki, M. et al. Aberrant gene methylation is a biomarker for the detection of cancer cells in peritoneal wash samples from advanced gastric cancer patients. Ann Surg Oncol 18, 3013-3019 (2011).

25 Ushiku, H. et al. DNA diagnosis of peritoneal fluid cytology test by CDO1 promoter DNA hypermethylation in gastric cancer. Gastric Cancer 20, 784-792 (2017).

26 McDonald, B. R. et al. Personalized circulating tumor DNA analysis to detect residual disease after neoadjuvant therapy in breast cancer. Sci Transl Med 11 (2019).

27 Leal, A. et al. White blood cell and cell-free DNA analyses for detection of residual disease in gastric cancer. Nat Commun 11, 525 (2020).

28 Abbosh, C. et al. Phylogenetic ctDNA analysis depicts early-stage lung cancer evolution. Nature 545, 446-451 (2017).

29 Azad, T. D. et al. Circulating Tumor DNA Analysis for Detection of Minimal Residual Disease After Chemoradiotherapy for Localized Esophageal Cancer. Gastroenterology 158, 494-505.e496 (2020).

30 Reinert, T. et al. Analysis of Plasma Cell-Free DNA by Ultradeep Sequencing in Patients With Stages I to III Colorectal Cancer. JAMA Oncol 5, 1124-1131 (2019).

31 Christensen, E. et al. Early Detection of Metastatic Relapse and Monitoring of Therapeutic Efficacy by Ultra-Deep Sequencing of Plasma Cell-Free DNA in Patients With Urothelial Bladder Carcinoma. J Clin Oncol 37, 1547-1557 (2019).

$32 \mathrm{Qu}, \mathrm{C}$. et al. Detection of early-stage hepatocellular carcinoma in asymptomatic HBsAg-seropositive individuals by liquid biopsy. Proc Natl Acad Sci U S A 116, 6308-6312 (2019).

33 Patel, J. P. et al. Prognostic relevance of integrated genetic profiling in acute myeloid leukemia. $N$ Engl J Med 366, 1079-1089 (2012).

34 Schuurhuis, G. J. et al. Minimal/measurable residual disease in AML: a consensus document from the European LeukemiaNet MRD Working Party. Blood 131, 1275-1291 (2018).

35 Thomassen, I. et al. Peritoneal carcinomatosis of gastric origin: a population-based study on 
incidence, survival and risk factors. Int J Cancer 134, 622-628 (2014).

36 Jung, Y. J., Seo, H. S., Kim, J. H., Park, C. H. \& Lee, H. H. Cross-Sectional Location of Gastric Cancer Affects the Long-Term Survival of Patients as Tumor Invasion Deepens. Ann Surg Oncol 24, 3947-3953 (2017).

37 La Torre, M. et al. Peritoneal wash cytology in gastric carcinoma. Prognostic significance and therapeutic consequences. Eur J Surg Oncol 36, 982-986 (2010).

38 Coccolini, F. et al. Peritoneal carcinomatosis. World J Gastroenterol 19, 6979-6994 (2013).

39 Katsuragi, K. et al. Prognostic impact of PCR-based identification of isolated tumour cells in the peritoneal lavage fluid of gastric cancer patients who underwent a curative RO resection. $\mathrm{Br} J$ Cancer 97, 550-556 (2007).

40 Bentrem, D., Wilton, A., Mazumdar, M., Brennan, M. \& Coit, D. The value of peritoneal cytology as a preoperative predictor in patients with gastric carcinoma undergoing a curative resection. Ann Surg Oncol 12, 347-353 (2005).

41 Hiraki, M. et al. Aberrant gene methylation in the peritoneal fluid is a risk factor predicting peritoneal recurrence in gastric cancer. World J Gastroenterol 16, 330-338 (2010).

42 Yonemura, Y. et al. Diagnostic value of preoperative RT-PCR-based screening method to detect carcinoembryonic antigen-expressing free cancer cells in the peritoneal cavity from patients with gastric cancer. ANZ J Surg 71, 521-528 (2001).

43 Coombes, R. C. et al. Personalized Detection of Circulating Tumor DNA Antedates Breast Cancer Metastatic Recurrence. Clin Cancer Res 25, 4255-4263 (2019).

44 Dudley, J. C. et al. Detection and Surveillance of Bladder Cancer Using Urine Tumor DNA. Cancer Discov 9, 500-509 (2019).

45 Razavi, P. et al. High-intensity sequencing reveals the sources of plasma circulating cell-free DNA variants. Nat Med 25, 1928-1937 (2019).

$46 \mathrm{Li}, \mathrm{J} . \mathrm{K}$. et al. Peritoneal lavage cytology and carcinoembryonic antigen determination in predicting peritoneal metastasis and prognosis of gastric cancer. World J Gastroenterol 11, 7374-7377 (2005).

47 Oyama, K., Terashima, M., Takagane, A. \& Maesawa, C. Prognostic significance of peritoneal minimal residual disease in gastric cancer detected by reverse transcription-polymerase chain reaction. Br J Surg 91, 435-443 (2004).

48 Leake, P. A. et al. A systematic review of the accuracy and utility of peritoneal cytology in patients with gastric cancer. Gastric Cancer 15 Suppl 1, S27-37 (2012).

49 Zhang, W. et al. Genetic Features of Aflatoxin-Associated Hepatocellular Carcinoma. Gastroenterology 153, 249-262.e242 (2017).

50 Fujikura, K. et al. Multiregion whole-exome sequencing of intraductal papillary mucinous neoplasms reveals frequent somatic KLF4 mutations predominantly in low-grade regions. Gut (2020). 
patients.

Fig. 1 Patient enrollment, sample detection workflow and the prognosis prediction of patients.

110 Gastric Cancer patients scheduled for surgery

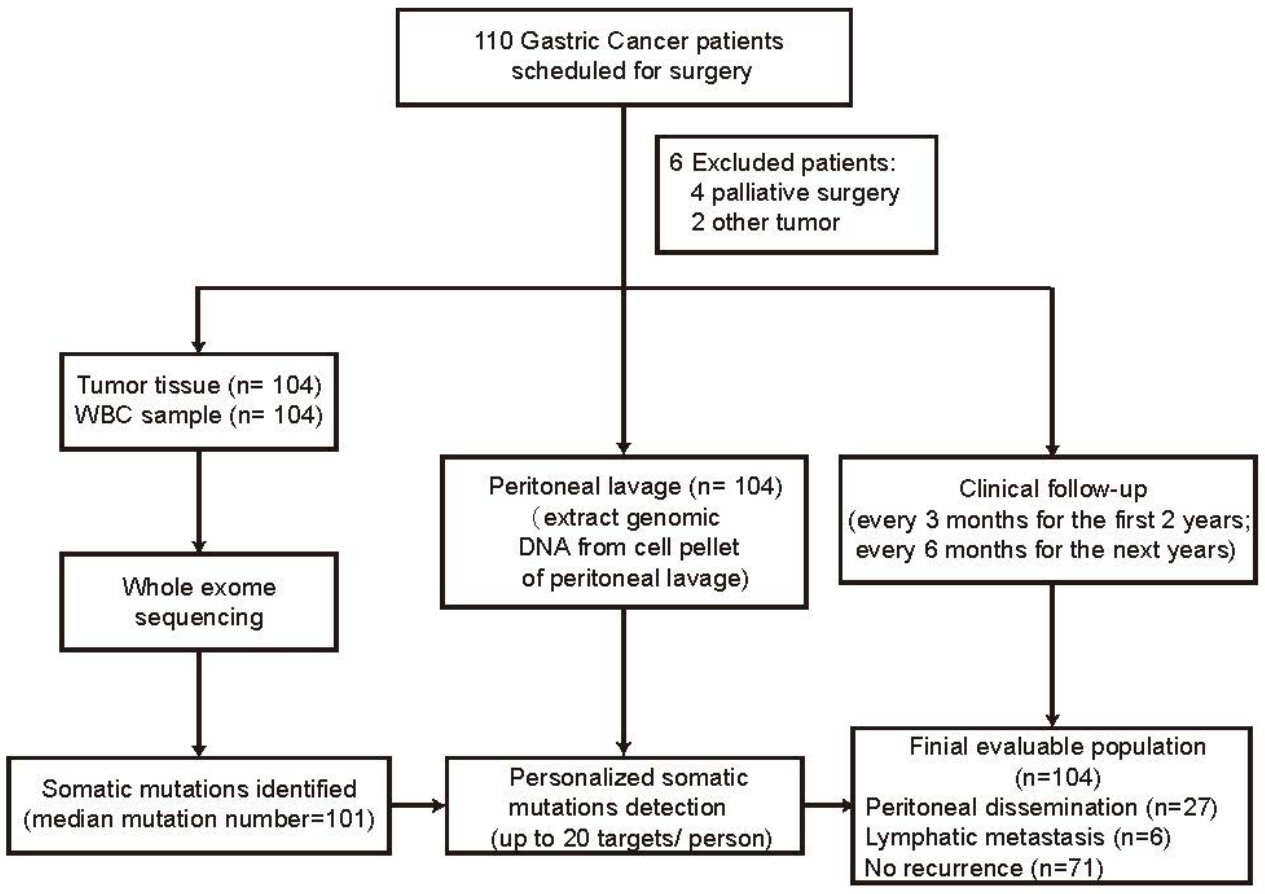



A3 mutant molecules
in D3 total molecules

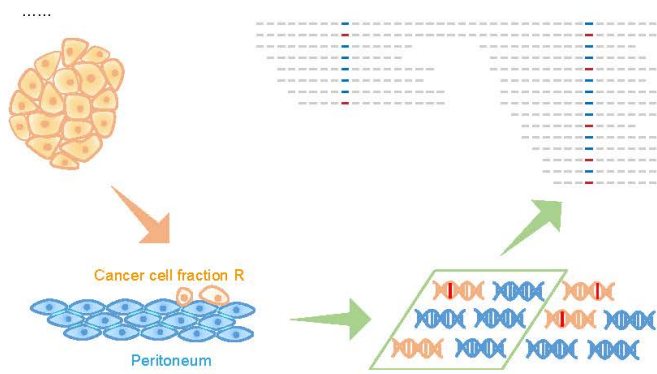

b

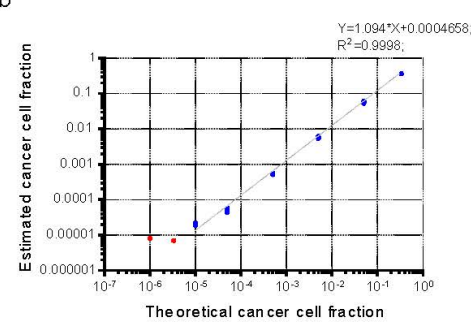

d
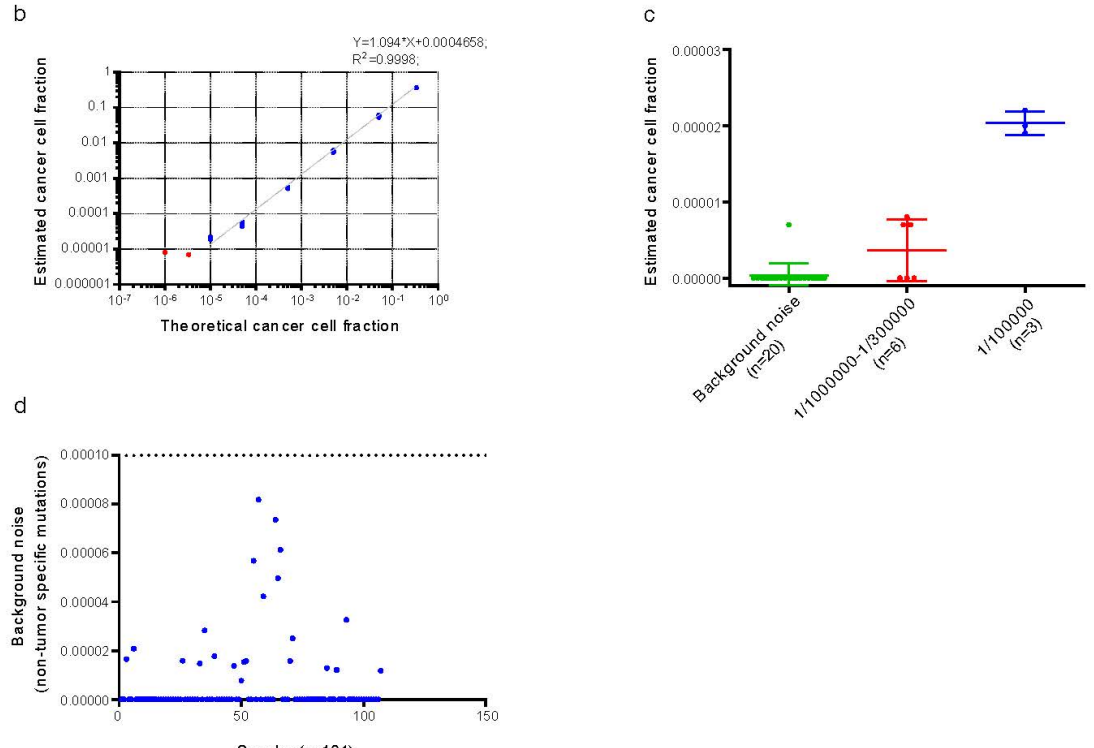

589

590

(a). Cancer cell fraction model. A model to estimate the cancer cell fraction based on allele frequency and sequencing depth of somatic mutations in tumor tissue and paired PLF samples. MAF: mutant allele frequency; $P t_{i}$ : MAF in solid tumor tissue; $P c_{i}$ : MAF in corresponding peritoneal lavage fluid (PLF); $D_{i}$ : sequencing depth in PLF; $A_{i}$ : mutation reads number in PLF; $X_{i}$ : observing reads with mutation in PLF; and $\mathrm{R}$ : overall cancer cell concentration. (b). The linear correlation between theoretical and estimated cancer cell fraction up to the dilution of $1: 10^{-5}$. Each dilution was repeated three times. The blue dots highlight the fractions above the limit of detection (PLC/PRF/5 cell fraction $=0.001 \%, 0.005 \%, 0.05 \%, 0.5 \%, 5 \%$ and $33 \%$ ). The red dots highlight the fractions under the limit of detection (PLC/PRF/5 cell fraction $=0.0001 \%, 0.0003 \%$ ).

600 (c). Background noise observed in the cancer cell fraction model at $\% \%$ PLC/PRF/5 cell input among the 20 independent replicates (green dots). Experiments performed on different cell line dilutions $(0.0001 \%$ and $0.0003 \%$, red dots; $0.001 \%$, blue dots) were repeated three times. (d). Biological noise of the 104 PLF samples from patients. The cancer cell fraction for each sample was calculated based on non-tumor-specific mutations. 
Fig. 3 Summary of clinical and histopathologic parameters, somatic mutations and cancer cell fraction for all patients.

Fig. 3 Summary of clinical and histopathologic parameters, somatic mutations and cancer cell fraction for all patients.

a

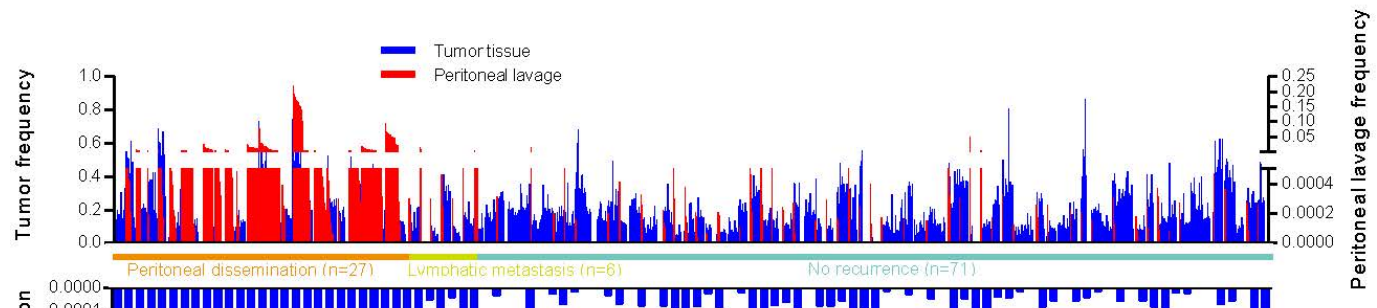

b
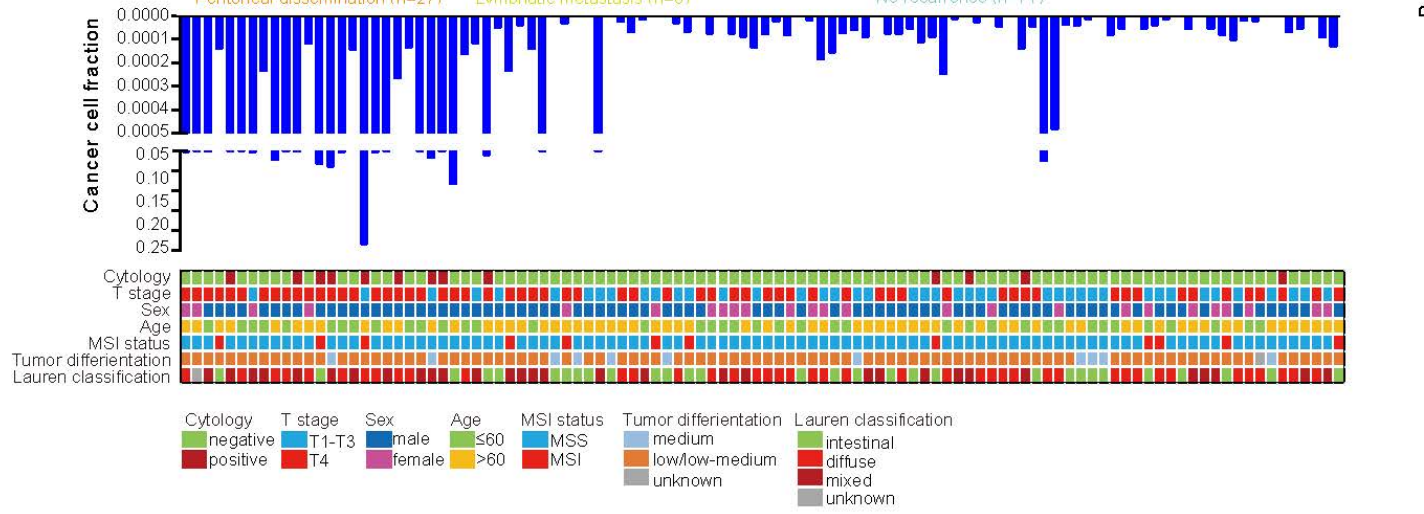

- Peritoneal dissemination

- Lymphatic metastasis

- No recurrence

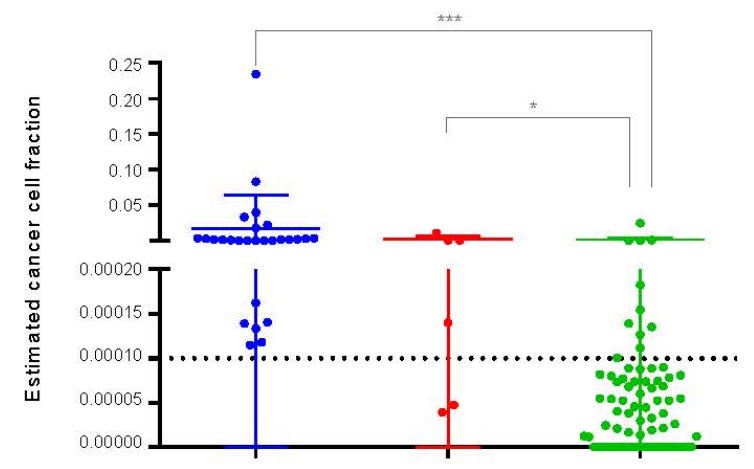

(a). Top panel, the summary of the frequencies of the tracked mutations in tumor and matched peritoneal lavage fluid samples from 104 patients. Blue bar, the tumor frequency of each tracked mutation. Frequency values are shown on the left vertical axis. Red bar, the detected peritoneal lavage fluid frequency of each tracked mutation. Frequency values are shown on the right vertical axis. The clinical outcome of patients is indicated under the bar. Middle panel, the summary of the cancer cell fractions for each patient. Bottom panel, clinical and histopathological characteristics.

(b). The cancer cell fraction distribution in patients with peritoneal dissemination $(n=27)$, lymphatic metastasis $(n=6)$ or no recurrence $(n=71)$. Reported $\mathrm{p}$ values are computed using 2-tailed Wilcoxon Mann-Whitney $\mathrm{U}$ test.

${ }^{* * *}, p<0.001 ;{ }^{*}, p<0.05$ 
649

650

651

652

653

654

655

656

657

658

659

660

661

662

663

664

665

666

667

668

669

670

671

672

673

674

675

676

677

678

679

680

681

682

683

684

685

686

687

688

689

690

691

692

Fig. 4 Kaplan-Meier estimates of recurrence-free survival (RFS) and overall survival (OS) for gastric cancer patients.

Fig. 4 Kaplan-Meier estimates of recurrence-free survival (RFS) and overall survival (OS) for gastric cancer patients.
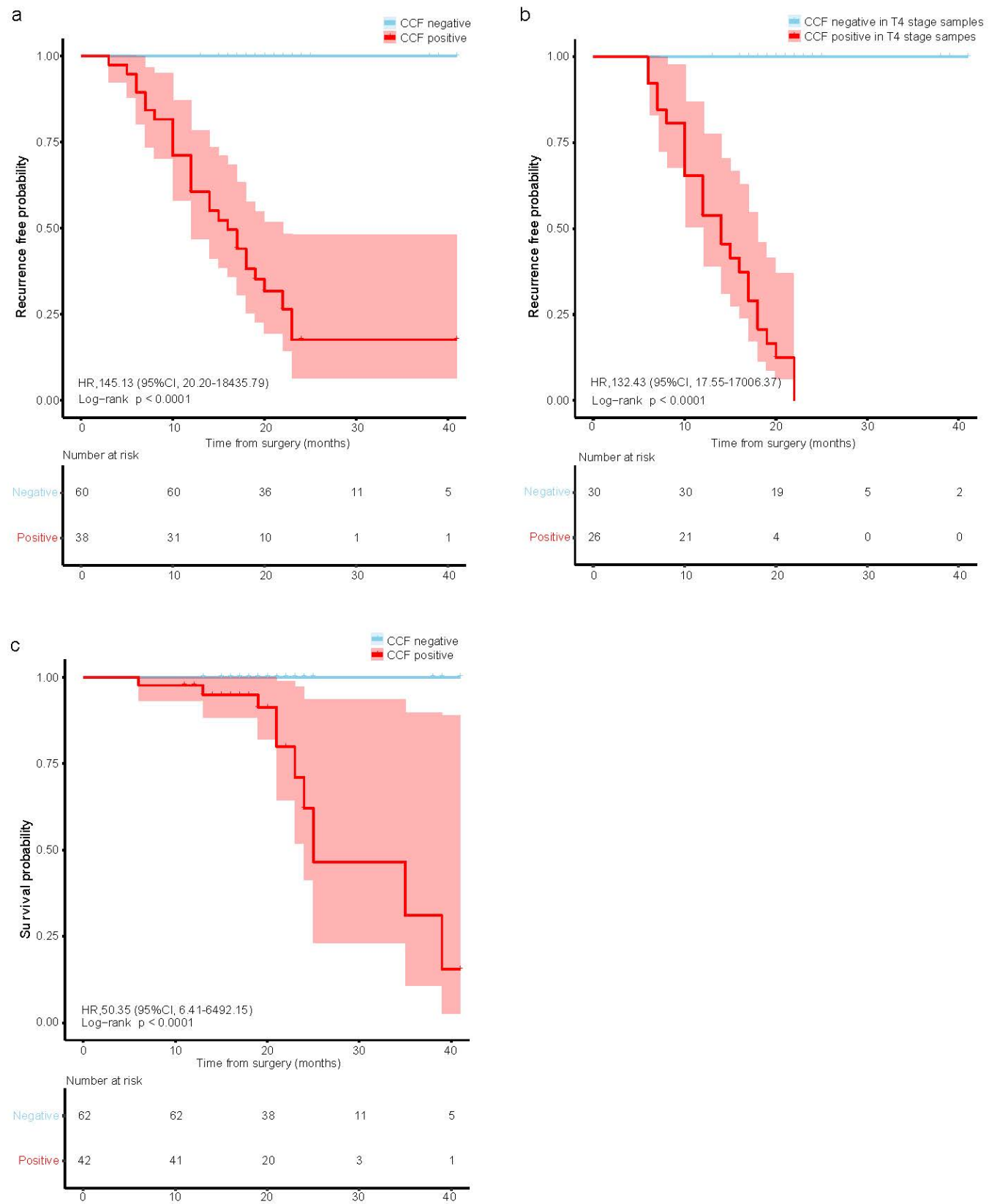

(a) and (b). Kaplan-Meier survival analysis shows probability of recurrence-free survival (RFS) as determined by A. cancer cell fraction detected in peritoneal lavage fluid $(n=98)$; and B. cancer cell fraction detected in peritoneal lavage fluid in stage T4 patients $(n=56)$ (for peritoneal dissemination). A patient was classified as testing positive if the cancer cell fraction detected in peritoneal lavage fluid was $\geq 0.01 \%$;

(c) Kaplan-Meier estimates of overall survival (OS) for 104 gastric cancer patients based on the estimated cancer cell fraction in peritoneal lavage fluid.

Shaded areas in the Kaplan-Meier plots indicate 95\%Cls. HR: hazard ratio; CCF: cancer cell fraction. 
Table 1 Binary results of the PLF mutation profiling model and clinical risk factors

\begin{tabular}{|c|c|c|c|c|c|c|c|}
\hline \multirow[b]{2}{*}{ Risk of } & & \multicolumn{2}{|c|}{ Estimated cancer cell } & \multicolumn{2}{|c|}{ Cytological } & \multicolumn{2}{|c|}{ Pathologic diagnosis } \\
\hline & & true PD & true noPD & true PD & true noPD & true PD & true noPD \\
\hline peritoneal & predict PD & 27 & 11 & 8 & 4 & 23 & 33 \\
\hline dissemination & predict noPD & 0 & 60 & 19 & 67 & 4 & 38 \\
\hline$(n=98)$ & Sensitivity & $100 \%$ & & $30 \%$ & & $85 \%$ & \\
\hline & Specificity & & $85 \%$ & & $94 \%$ & & $54 \%$ \\
\hline & PPV & $71 \%$ & & $67 \%$ & & $41 \%$ & \\
\hline & NPV & & $100 \%$ & & $78 \%$ & & $90 \%$ \\
\hline Risk of & & true RE & true noRE & true $\mathrm{RE}$ & true noRE & true RE & true noRE \\
\hline recurrence & predict RE & 31 & 11 & 9 & 4 & 28 & 33 \\
\hline$(n=104)$ & predict noRE & 2 & 60 & 24 & 67 & 5 & 38 \\
\hline & Sensitivity & $94 \%$ & & $27 \%$ & & $85 \%$ & \\
\hline & Specificity & & $85 \%$ & & $94 \%$ & & $54 \%$ \\
\hline & PPV & $74 \%$ & & $69 \%$ & & $46 \%$ & \\
\hline & NPV & & $97 \%$ & & $74 \%$ & & $88 \%$ \\
\hline
\end{tabular}

PD, peritoneal dissemination; RE, recurrence; PPV, positive predictive value; NPV, negative predictive value.

Estimated cancer cell fraction was defined as positive (CCF $\geq 0.01 \%$ ) or negative (CCF $<0.01 \%$ ).

Pathologic diagnosis was defined as high (T4) or low (T0-3) according to the standard criteria. 


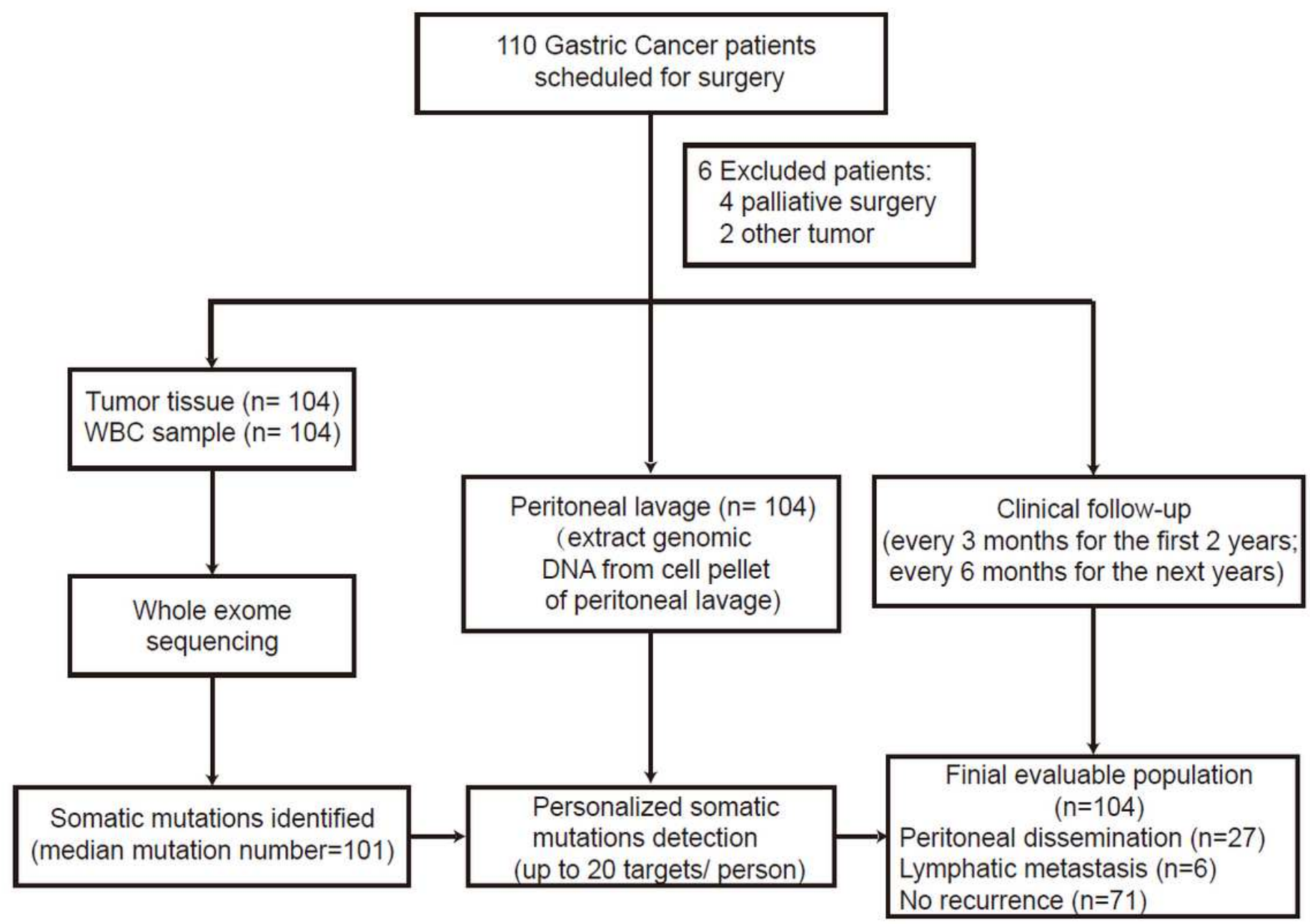

\section{Figure 1}

Patient enrollment, sample detection workflow and the prognosis prediction of patients. 


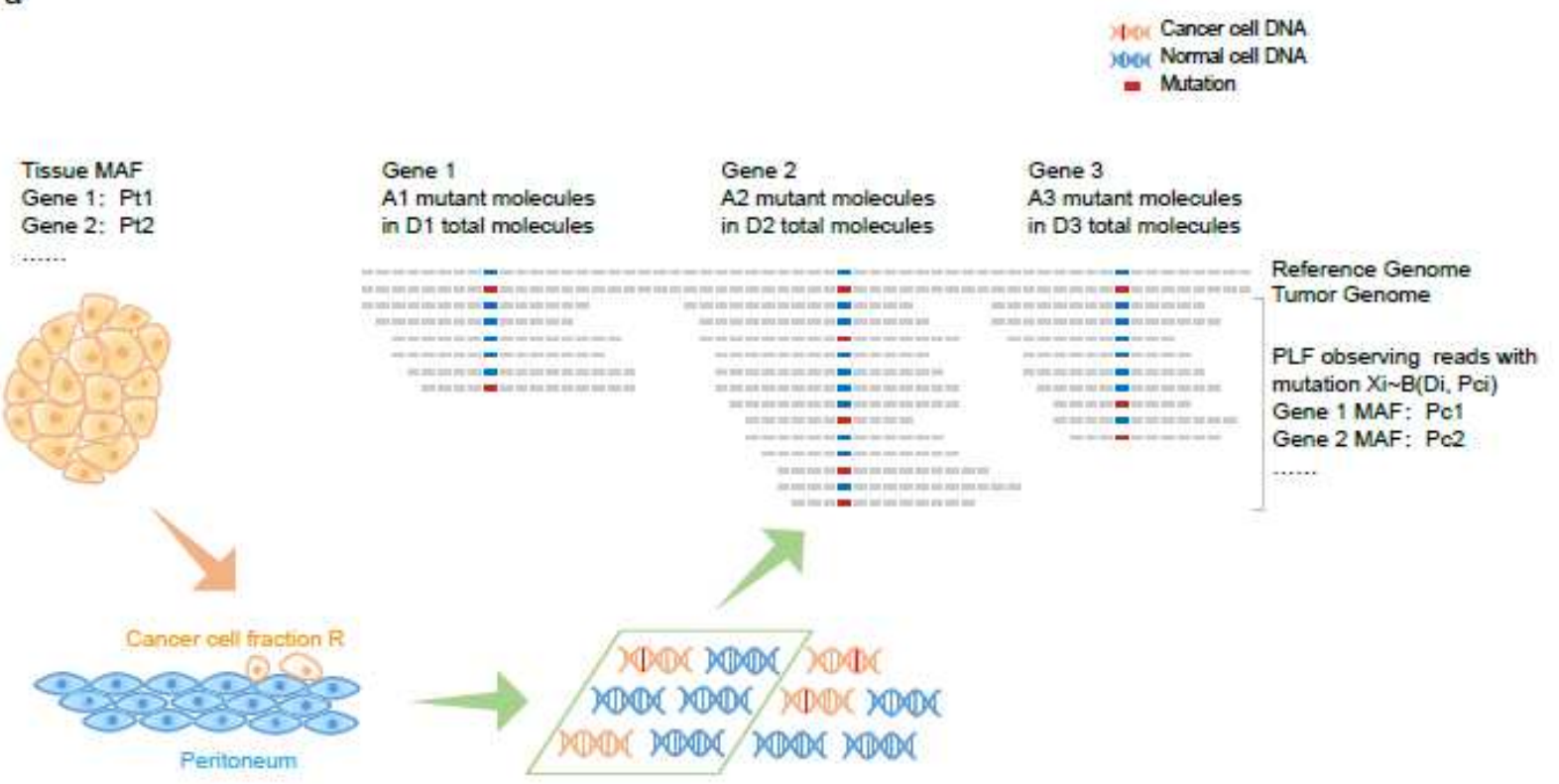

b

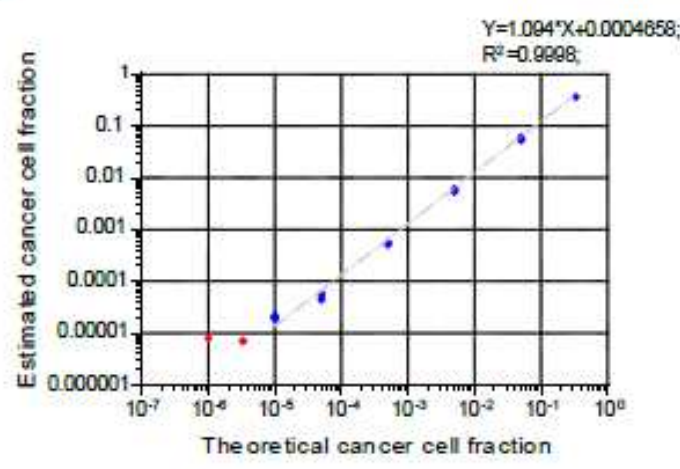

d

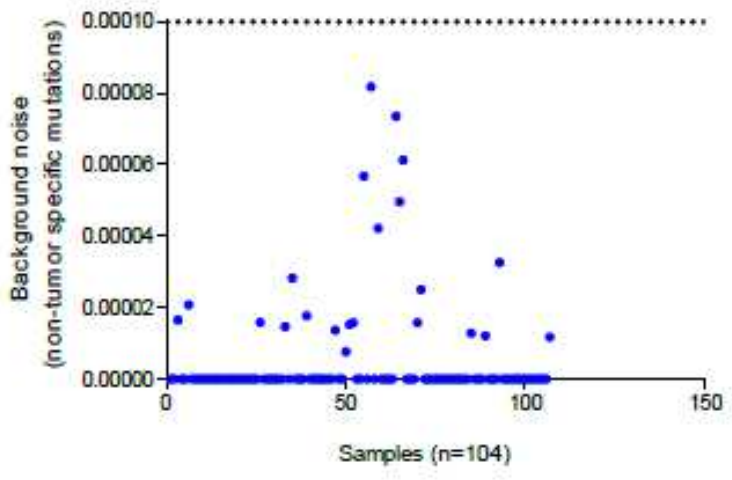

c

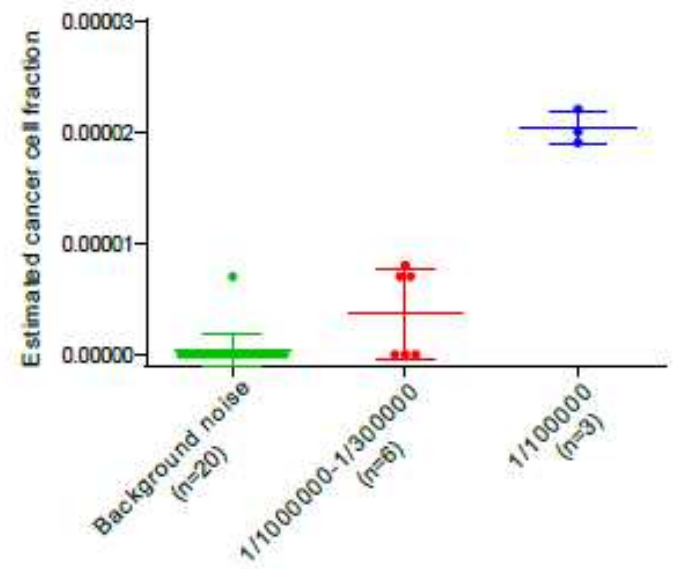

\section{Figure 2}

Cancer cell fraction model and background noise. (a). Cancer cell fraction model. A model to estimate the cancer cell fraction based on allele frequency and sequencing depth of somatic mutations in tumor

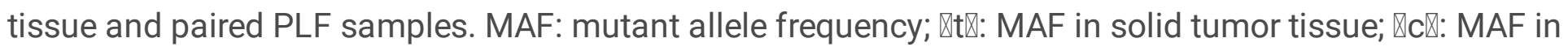
corresponding peritoneal lavage fluid (PLF); 怄: sequencing depth in PLF; $\mathbb{Q}$ : mutation reads number in PLF; 怄: observing reads with mutation in PLF; and R: overall cancer cell concentration. (b). The linear 
correlation between theoretical and estimated cancer cell fraction up to the dilution of 1:10-5. Each dilution was repeated three times. The blue dots highlight the fractions above the limit of detection $(\mathrm{PLC} / \mathrm{PRF} / 5$ cell fraction $=0.001 \%, 0.005 \%, 0.05 \%, 0.5 \%, 5 \%$ and $33 \%)$. The red dots highlight the fractions under the limit of detection (PLC/PRF/ 5 cell fraction $=0.0001 \%, 0.0003 \%)$. (c). Background noise observed in the cancer cell fraction model at $0 \% \mathrm{PLC} / \mathrm{PRF} / 5$ cell input among the 20 independent replicates (green dots). Experiments performed on different cell line dilutions $(0.0001 \%$ and $0.0003 \%$, red dots; $0.001 \%$, blue dots) were repeated three times. (d). Biological noise of the 104 PLF samples from patients. The cancer cell fraction for each sample was calculated based on non-tumor-specific mutations.

a

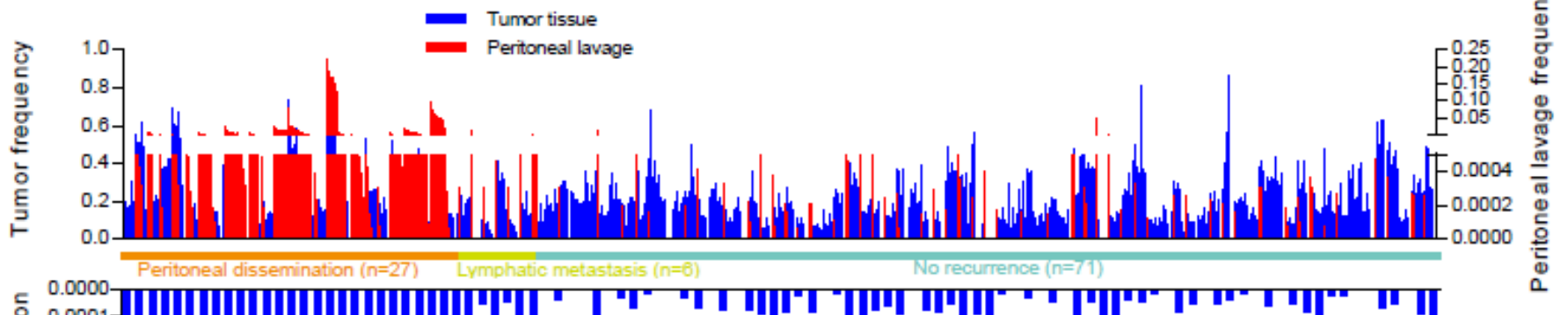

b

- Peritoneal dissemination

- Lymphatic metastasis

- No recurrence

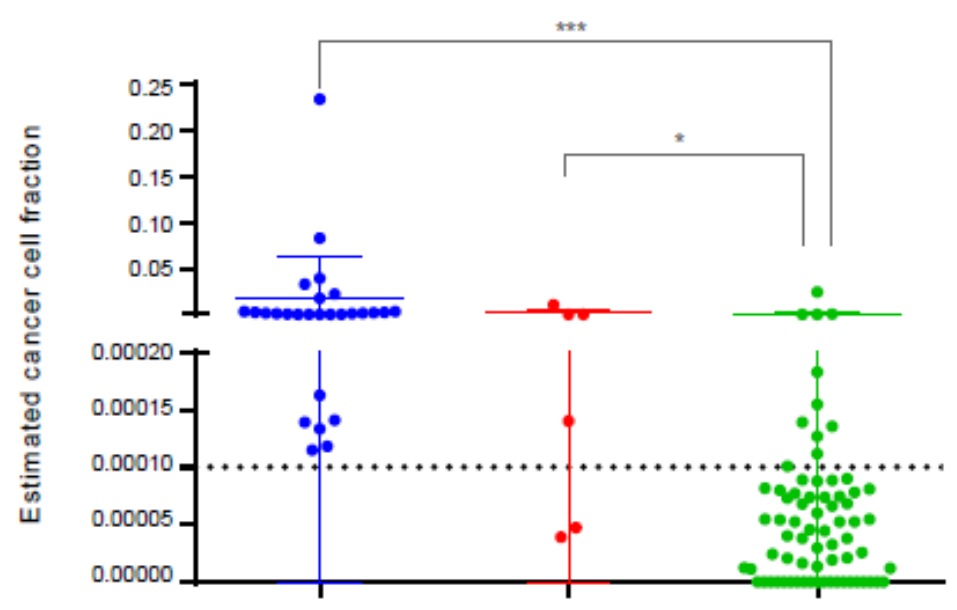




\section{Figure 3}

Summary of clinical and histopathologic parameters, somatic mutations and cancer cell fraction for all patients. (a). Top panel, the summary of the frequencies of the tracked mutations in tumor and matched peritoneal lavage fluid samples from 104 patients. Blue bar, the tumor frequency of each tracked mutation. Frequency values are shown on the left vertical axis. Red bar, the detected peritoneal lavage fluid frequency of each tracked mutation. Frequency values are shown on the right vertical axis. The clinical outcome of patients is indicated under the bar. Middle panel, the summary of the cancer cell fractions for each patient. Bottom panel, clinical and histopathological characteristics. (b). The cancer cell fraction distribution in patients with peritoneal dissemination $(n=27)$, lymphatic metastasis $(n=6)$ or no recurrence $(n=71)$. Reported $p$ values are computed using 2-tailed Wilcoxon Mann-Whitney $U$ test. ***, $p<0.001 ; *, p<0.05$ 

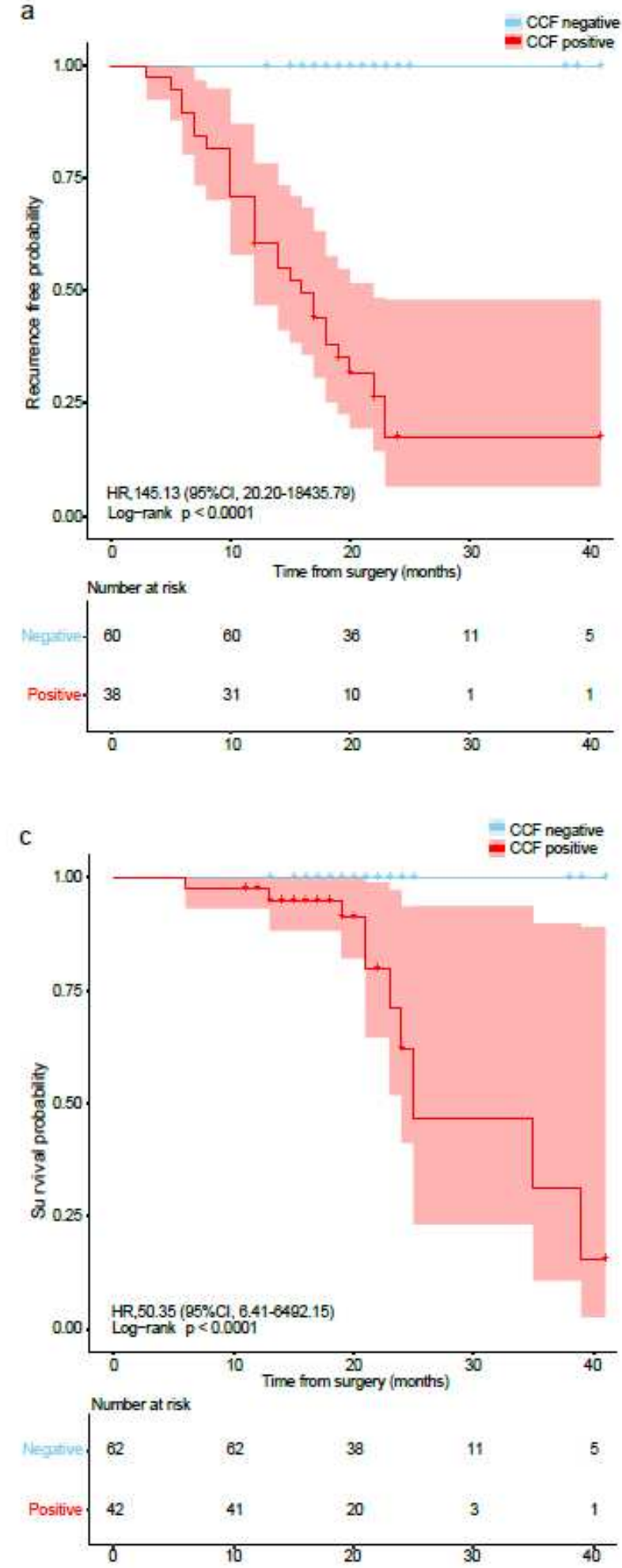

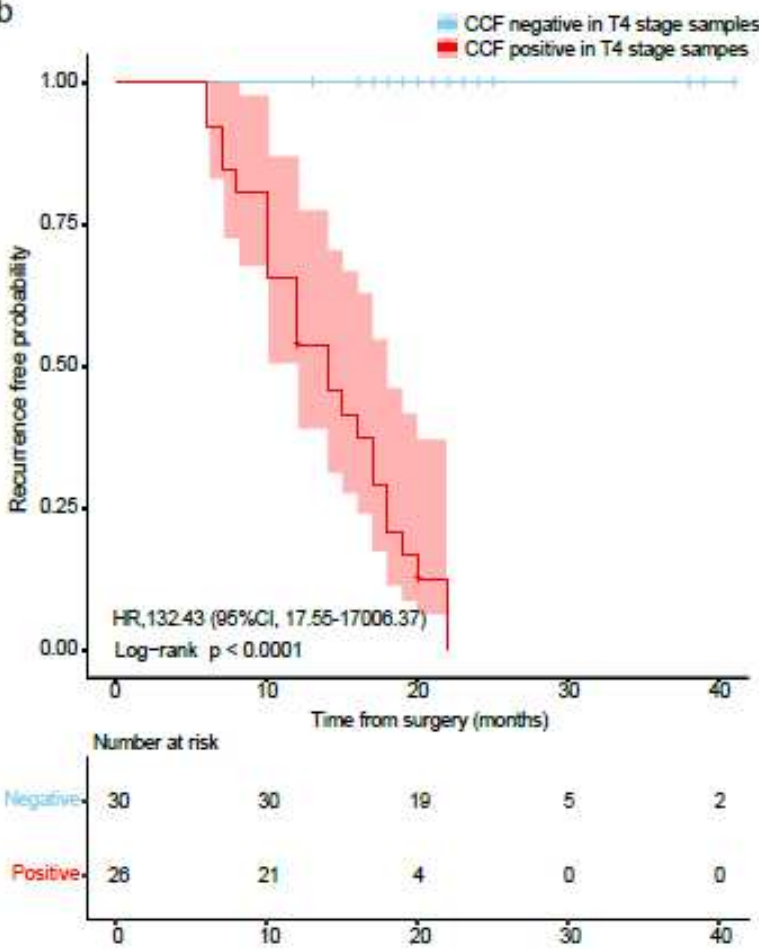

\section{Figure 4}

Kaplan-Meier estimates of recurrence-free survival (RFS) and overall survival (OS) for gastric cancer patients. (a) and (b). Kaplan-Meier survival analysis shows probability of recurrence-free survival (RFS) as determined by $A$. cancer cell fraction detected in peritoneal lavage fluid $(n=98)$; and B. cancer cell fraction detected in peritoneal lavage fluid in stage T4 patients $(n=56)$ (for peritoneal dissemination). A patient was classified as testing positive if the cancer cell fraction detected in peritoneal lavage fluid was 
$>0.01 \%$; (c) Kaplan-Meier estimates of overall survival (OS) for 104 gastric cancer patients based on the estimated cancer cell fraction in peritoneal lavage fluid. Shaded areas in the Kaplan-Meier plots indicate 95\%Cls. HR: hazard ratio; CCF: cancer cell fraction.

\section{Supplementary Files}

This is a list of supplementary files associated with this preprint. Click to download.

- SupplementaryFigures.pdf

- SupplementaryTables.xlsx 